PHYSICAL REVIEW D 90, 103003 (2014)

\title{
Describing the observed cosmic neutrinos by interactions of nuclei with matter
}

\author{
Walter Winter \\ DESY, Platanenallee 6, 15738 Zeuthen, Germany \\ (Received 1 August 2014; published 11 November 2014)
}

\begin{abstract}
IceCube has observed neutrinos that are presumably of extra-Galactic origin. Since specific sources have not yet been identified, we discuss what could be learned from the conceptual point of view. We use a simple model for neutrino production from the interactions between nuclei and matter, and we focus on the description of the spectral shape and flavor composition observed by IceCube. Our main parameters are the spectral index, maximal energy, magnetic field, and composition of the accelerated nuclei. We show that a cutoff at PeV energies can be achieved by soft enough spectra, a cutoff of the primary energy, or strong enough magnetic fields. These options, however, are difficult to reconcile with the hypothesis that these neutrinos originate from the same sources as the ultrahigh-energy cosmic rays. We demonstrate that heavier nuclei accelerated in the sources may be a possible way out if the maximal energy scales appropriately with the mass number of the nuclei. In this scenario, neutrino observations can actually be used to test the ultrahigh-energy cosmic ray acceleration mechanism. We also emphasize the need for a volume upgrade of the IceCube detector for future precision physics, for which the flavor information becomes a statistically meaningful model discriminator as well as a qualitatively new ingredient.
\end{abstract}

DOI: $10.1103 /$ PhysRevD.90.103003

PACS numbers: 95.85.Ry, 98.70.Sa, 95.55.Vj

\section{INTRODUCTION}

One of the major breakthroughs in astronomy has been the discovery of high-energy neutrinos by the IceCube detector at the South Pole. The story started with the announcement of two PeV neutrino events in 2012 [1]. As a consequence of changing the search strategy, over 28 events with deposited energies greater than $30 \mathrm{TeV}$ were announced in 2013 [2], and 37 events were recorded in the current three-year data analysis [3]. While about 15 of these 37 events are expected to be from the backgrounds of atmospheric muons and neutrinos, the excess over the background currently constitutes more than $5 \sigma$. Clearly, high-energy neutrino astronomy is emerging as a new discipline with IceCube at the forefront, which is expected to collect 100-200 events (within the current analysis scheme) during its lifetime. While this is significant statistics, it may not be sufficient for resolving individual sources [4] and for precision studies of spectrum and flavor composition. Therefore, next-generation experiments are being discussed, such as a volume upgrade of the IceCube detector [the IceCube high-energy extension (HEX)] and the $\mathrm{KM} 3 \mathrm{NeT}$ experiment in the Mediterranean [5]. The results of the IceCube experiment during this and the coming years will have to serve as input for the optimization of these future options.

On the theoretical side, there have been many speculations regarding where these neutrinos would be coming from; see, e.g., Ref. [6] for a recent review. It is probably fair to say that there is no general answer to this question yet. Instead, the current state of the art can be recast in a number of conceptual questions: (i) What is the role of atmospheric neutrinos (in particular, prompt neutrinos)?

(ii) Are there any directional or time-wise clusters, or is the flux isotropic? Are there any correlations with known objects or events?

(iii) Are some of the events of Galactic origin?

(iv) Why are there no events above a few PeV?

(v) Can the neutrinos stem from the sources of the ultrahigh-energy cosmic rays (UHECRs)?

(vi) Are the observed neutrinos coming from one source class, or more than one? Which ones?

(vii) Is the flavor composition what is expected, or are there deviations indicative of either physics beyond the Standard Model or nonconventional compositions at the source?

(viii) Is there a particle physics origin of these neutrinos, such as dark matter?

In this study, we focus on the conceptual interpretation of the observed events in terms of the spectral shape of the observed flux. That is, we assume that the neutrinos come from one source population with similar properties as those of extra-Galactic origin, as there is not yet any evidence for directional clusters. We furthermore assume that the source population is cosmologically distributed such that it roughly follows the star formation rate. We postulate that the neutrinos are produced from interactions between nuclei and matter. This is, in a way, the simplest possible class of models, as the neutrino spectrum directly follows the nonthermal spectrum of the accelerated nuclei; for interactions with radiation, the obtained spectral shape of the neutrinos depends on both the spectra of the interacting nuclei and the target photons (see, e.g., Ref. [7] for target 
photons produced by synchrotron radiation of coaccelerated electrons). However, we do take magnetic-field effects on the secondary muons and pions into account, which can significantly alter the neutrino spectra and flavor composition. (See, e.g., Refs. [8-10], in particular in the context of gamma-ray bursts [11-13] and microquasars [14,15]; see Ref. [16] for a review.) While most of these studies discussed interactions between protons and photons, the secondaries produced by interactions between nuclei and matter will be affected by magnetic fields as well; see e.g., Ref. [14]. In the context of a possible cutoff at PeV energies in recent IceCube observations, magnetic-field effects on the secondaries may be a way to decouple the maximal proton from the maximal neutrino energy; see Ref. [7] for a more detailed discussion. Finally, there seems to be growing evidence for a heavier composition of the UHECRs [17]. We therefore take into account the composition of the nuclei in the sources. In particular, we discuss whether the cutoff at $\mathrm{PeV}$ neutrino energies can be consistent with the UHECR paradigm if heavier nuclei are accelerated to higher energies within the sources. In fact, we will demonstrate that one can learn something about the acceleration mechanism in this scenario. Note that the interpretation of the obtained neutrino-flux normalization in terms of source luminosity and column depth will be discussed elsewhere.

\section{MODEL AND METHODS}

The interaction model used in this work is based on the parametrization by Kelner et al. [18] for proton-proton interactions, where we take into account the charged-pion production explicitly to allow for secondary cooling. For the extension to heavier nuclei $A p$ interactions, see Ref. [19]. The secondary production $Q_{\pi}\left[\mathrm{cm}^{-3} \mathrm{~s}^{-1} \mathrm{GeV}^{-1}\right]$ is given from the nonthermal nucleon density in the source $N_{A}\left[\mathrm{~cm}^{-3} \mathrm{GeV}^{-1}\right]$ and the target nucleon density $n_{p}\left[\mathrm{~cm}^{-3}\right]$ by

$$
\begin{aligned}
Q_{\pi}\left(E_{\nu}\right)= & c n_{p} \int_{0}^{1} \sigma_{A p}\left(\frac{E_{\nu}}{x_{A}}\right) N_{A}\left(\frac{E_{\nu}}{x_{A}}\right) \\
& \times A f\left(A x_{A}, \frac{E_{\nu}}{A x_{A}}\right) \frac{d x_{A}}{x_{A}},
\end{aligned}
$$

where $x_{A}=x / A$ is the fraction of the nucleus' energy going into the neutrino, $f$ are the scaling functions from Ref. [18] (SIBYLL-based versions), and $\sigma_{A p}=A^{3 / 4} \times \sigma_{p p}$ [20]. If the target material is heavier than hydrogen, one may superimpose the nuclei $n_{p} \simeq A \times n_{A}$. However, there are corrections to this, but-since we do not discuss the normalization in this study - this does not affect our results. Note that Eq. (1) can be rewritten in terms of the column density $L n_{p}$, where the interpretation of $L$ in terms of the size of the interaction region depends on the scenario. For example, by injecting nuclei into the interaction region with
TABLE I. Main parameters of the model.

\begin{tabular}{lll}
\hline \hline Parameter & \multicolumn{1}{c}{ Description } & \multicolumn{1}{c}{ Unit } \\
\hline$\alpha$ & Spectral index of primary nuclei & None \\
$E_{\max }$ & Maximal energy & $\mathrm{GeV}$ \\
$B$ & Magnetic field & Gauss (G) \\
$A$ & Mass number & None \\
\hline \hline
\end{tabular}

the rate $Q_{A}$, one can estimate that $N_{A} \simeq Q_{A} t_{\text {esc }}$ in the absence of disintegration and cooling, and therefore $L n_{p}=$ $c t_{\text {esc }} n_{p}$ is the column density, which determines the normalization of Eq. (1). The pion and consequent muon decays are computed in the usual way, including the helicity dependence of the muon decays; see Ref. [10]. Flavor mixing is taken into account with the best-fit values from Ref. [21] (first octant solution).

The main parameters of the model are listed in Table I. We start with $N_{A}(E) \propto E^{\alpha} \exp \left(-E / E_{\max }\right)$ in Eq. (1), where $\alpha$ is the initial spectral index, which is expected to be $\alpha \sim 2$ from Fermi shock acceleration. The maximal energy $E_{\max }$ is typically obtained by equating the acceleration time scale with the dominant escape and energy loss time scales in a specific scenario. Since this derivation is highly model dependent, we keep $E_{\max }$ as a model parameter. Note that $E_{\max }$ can also be used to simulate a spectral break in the initial spectrum, as it may come from an energy-dependent escape time frequently discussed for starburst galaxies [22]; see also Refs. [23-25]. In addition, in Eq. (1) $N_{A}$ already corresponds to the result including disintegration and other cooling and escape processes. This means that $E_{\max }$ could also describe a spectral break from a cooling process, an energy-dependent escape, or photo-disintegration. The secondary muons and pions are assumed to undergo synchrotron losses and decays governed by the magnetic field $B$, which impacts the spectral shape and flavor composition; see Refs. [10,13]. Note that there could be other cooling or escape processes affecting the secondaries, such as adiabatic cooling (see, e.g., Ref. [26]) or reacceleration [27-29]. These effects are, however, model dependent and typically not the dominant ones shaping the neutrino spectra. Finally, we have the composition $A$ as a parameter. In fact, Eq. (1) allows to use an energydependent (average) composition $A\left(E_{A}\right)=A\left(E_{\nu} / x_{A}\right)$, which we will use below. Note that for a power law with $\alpha=-2$, the composition would not affect our results. However, both the cutoff and the varying composition will change this conclusion.

For the sake of simplicity, we furthermore assume that the sources do not have large Doppler factors, and that they are cosmologically distributed following the star formation rate by Hopkins and Beacom [30], i.e, $\quad E^{2} \phi_{\nu} \propto \int_{z} Q_{\nu}(E(1+z)) H(z) d V / d z\left(4 \pi d_{L}^{2}\right)^{-2} d z$ for steady sources, where $H(z)$ is the source density normalized to the local source density $[H(0) \equiv 1], \Omega_{m}=0.27$, 
and $\Omega_{\Lambda}=0.73$. For transients, there will be another factor $1+z$ in the denominator of the integrand; we find, however, that the results depend very little on the details of the cosmological source distribution.

For the fit, we follow Ref. [7], using the up-to-date threeyear data from Ref. [3]. We use eight bins: four in the reconstructed neutrino energy (30-200 TeV, $200 \mathrm{TeV}$ to $1 \mathrm{PeV}, 1-3 \mathrm{PeV}$, and 3-100 PeV) and one bin for muon tracks and cascades within each energy slot. For the sake of simplicity, we assume that the electromagnetic equivalent energy is roughly $25 \%$ of the incident neutrino energy for a muon track, and $75 \%$ for a cascade [31]; cascades from neutral-current interactions are assumed to be suppressed by the cross sections, the lower fiducial mass (see Fig. 7 in Ref. [2]), and the fact that only a fraction of the initial neutrino energy is deposited in the detector [1]. The (Poissonian) $\chi^{2}$ is obtained by bin-wise comparison of the 36 observed events (for which energy information is available) with the prediction. The prediction with a free overall normalization is obtained from folding the three flavored neutrino fluxes for one set of model parameters with the corresponding exposures derived from the effective areas in Ref. [2]. Then the atmospheric backgrounds are added. The atmospheric neutrino background is derived from the IceCube observation of muon neutrinos [32,33], and for the atmospheric muons we assume the same shape. The measurement of the atmospheric electron neutrino background is much more uncertain. We therefore extrapolate it from the muon neutrino background, which is roughly consistent with the flavor composition in Ref. [34]. We obtain 3.6 background muon tracks from neutrinos, 3.2 cascades from neutrinos, and 8.6 muon tracks from atmospheric muons, matching the publicly available information on the IceCube analysis. This means that the predicted number of muon tracks is slightly higher than the observation (eight), a fact which was discussed in detail in Ref. [35] (see also Ref. [36] for some discussion). While this unavoidable tension increases the minimal $\chi^{2}$ of our fit, it hardly affects the $\Delta \chi^{2}$, and therefore it is of little relevance for the results in this study. As a final step the $\chi^{2}$ between the predicted and observed rates are summed over all bins and minimized over the free normalization of the astrophysical flux to obtain the best fit for the chosen parameters.

\section{RESULTS FOR PROTON-MATTER INTERACTIONS}

Here we first reproduce the IceCube spectral fit in Ref. [3], which is based on a subsample of the 37 events with $60 \mathrm{TeV} \leq E_{\mathrm{dep}} \leq 3 \mathrm{PeV}$. All of the following figures are based on the full data sample. In Fig. 1, the allowed fit regions (filled contours) are shown for a three-parameter $\left(\alpha, B, E_{\max }\right)$ model for protons only, where one of the parameters is fixed in each panel. Most noteworthy, for $B \leq 10^{2} \mathrm{G}$ we obtain a spectral index $\alpha=-2.3 \pm 0.3$, which is consistent with the IceCube Collaboration's results. Thus, although our procedure qualitatively deviates from internal analyses of the IceCube Collaboration in a few ways (e.g., mapping from deposited to reconstructed energy/energy reconstruction, details on the background model, and systematic errors), we can roughly reproduce their results in order to test more complicated models. For instance, one can read off from this figure that $E_{\max }$ and $B$ can produce a cutoff as an alternative to softer spectra. For instance, for a strong enough $B$ a spectral index $\alpha=-2$ is allowed. Note that in the following we will use the full set of events to make full use of the statistics.

An interesting observation was made by Murase, Ahlers, and Lacki (MAL) [37]: the production of gamma rays from $\pi^{0}$ decays in the sources-which are coproduced with the charged pions-may violate the Fermi isotropic background measurements [38]. The highest-energy data points are at about $100 \mathrm{GeV}$, which is significantly below the measured neutrino energies and requires some extrapolation of the spectrum. In addition, gamma rays may come from higher energies via the initiated electromagnetic cascade. While the details are somewhat model dependent, we compute the gamma-ray flux injected at the sources based on Ref. [18]. We add a penalty $\chi^{2}$, imposing that $\left.E^{2} \phi_{\gamma}\right|_{100 \mathrm{GeV}}=8_{-\infty}^{+2} 10^{-8} \mathrm{GeV} \mathrm{cm}^{-2} \mathrm{~s}^{-1} \mathrm{sr}^{-1}$, i.e., an upper bound. The effect of this penalty is depicted by the dashed curves in Fig. 1; it leads to a lower cutoff $\alpha \gtrsim-2.2(1 \sigma)$. This result is consistent with Ref. [37], where $\alpha \gtrsim-2.18$ was found. Note that for the chosen star formation evolution of the sources, the main contribution will come from $z \sim 1$, where the optical depth at $100 \mathrm{GeV}$ is still small enough that most gamma rays can reach us without being attenuated in photon background fields during their propagation. However, the gamma-ray constraint can be at least partially avoided if a spectral break or lower cutoff in the energy spectrum of the nonthermal nucleons is introduced. We therefore discuss it separately in this study.

Let us now take into account all 36 events with energy information. The fit result is shown in Fig. 2 for a proton composition, where one of the parameters is fixed in each panel (parameter space "section"). The minimal $\chi^{2}$ is about 9 in the left-hand panels, and the $\chi^{2} /$ d.o.f. is about 2 . This relatively large value comes from the above-mentioned tension between the muon track prediction and observation, and can in principle be avoided by using a different background model. However, given the small number of bins (eight), it should not be overemphasized. Comparing to Fig. 1, we note that the spectral index $\alpha$ shifts to softer values $(\alpha=-2.7 \pm 0.2,1 \sigma$ for 1 d.o.f.); see the upper left panel for small $B$. Apart from a better matching at low energies, the information beyond $3 \mathrm{PeV}$ leads to stronger constraints because no neutrinos have been seen there. However, while there is a tendency towards softer spectra $\alpha \ll-2.3$ (for the best fit) in all tested cases, the exact bestfit value of $\alpha$ depends on details of how the (steep) 

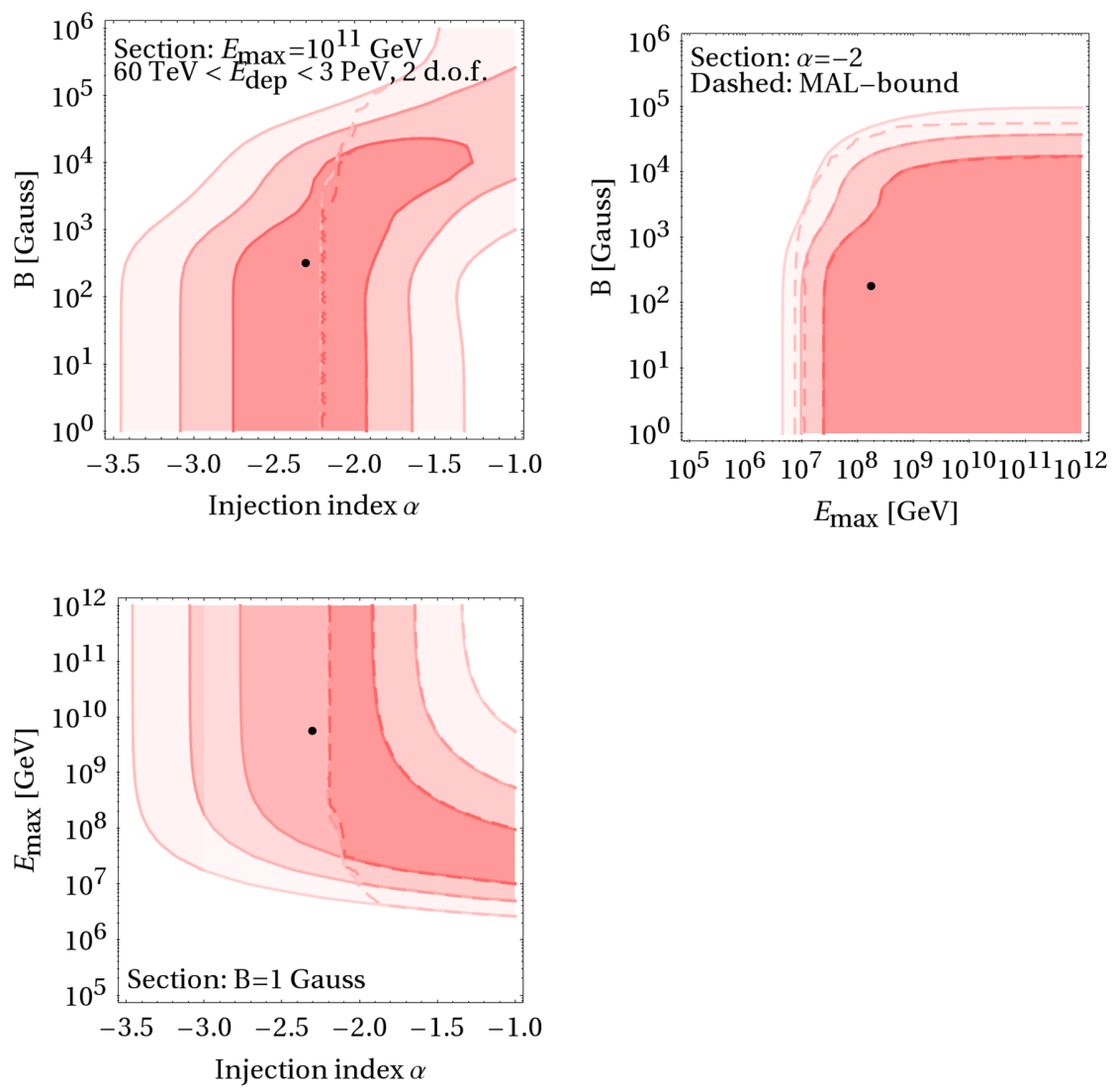

FIG. 1 (color online). Allowed fit regions to neutrino data at $1 \sigma, 2 \sigma$, and $3 \sigma$ (two d.o.f.) in a three-parameter $\left(\alpha, B, E_{\max }\right)$ model for protons only, where one of the parameters is fixed in each panel (sections shown). Only events used by the IceCube Collaboration fit [3] in terms of the deposited energy $60 \mathrm{TeV} \leq E_{\mathrm{dep}} \leq 3 \mathrm{PeV}$ have been used. Dashed contours refer to including a generic bound by MAL [37] on the $\gamma$-ray emission from $\pi^{0}$ 's coproduced with charged pions if the spectrum extends down to $100 \mathrm{GeV}$, where it has to obey the Fermi isotropic background bound [38]. The best fit (for the solid contours) is marked by the dot.

atmospheric backgrounds are implemented. We also have a clear limit in the $E_{\max }-B$ plane (upper right panel), where the lower right corner is excluded because it would produce too many high- $E$ events. In that panel, two distinctive regions appear at the $1 \sigma$ confidence level: one produces the cutoff with $10^{7} \mathrm{GeV} \lesssim E_{\max } \lesssim 10^{8} \mathrm{GeV}$, while the other produces the cutoff with $B \sim 10^{4} \mathrm{G}$. It is noteworthy that these regions can be potentially discriminated by the flavor composition of the neutrinos: roughly on the rhs of the dash-dotted line, the neutrino production will be dominated by pion decays at the highest energies, whereas the muons lose energy faster than they decay ("muon-damped source"). As a consequence, only muon neutrinos and antineutrinos will be produced at the source, which leads to a deviation from the canonical $\left(\nu_{e}: \nu_{\mu}: \nu_{\tau}\right) \sim(1: 1: 1)$ flavor composition at the detector including flavor mixing [39]; see Ref. [16] for a review.

A potential theoretical constraint comes from proton synchrotron losses: not necessarily all of the regions shown in Fig. 2 can be reached, as protons may lose energy in magnetic fields faster than they can be accelerated. This can be quantified using an acceleration rate for shock acceleration [40],

$$
t_{\mathrm{acc}}^{-1}=\eta \frac{c^{2} Z e B}{E},
$$

which corresponds to a constant fractional energy gain per cycle $\eta$. For efficient acceleration, one typically assumes $\eta \simeq 1$. Synchrotron losses are governed by

$$
t_{\text {synchr }}^{-1}=\frac{Z^{4} e^{4} B^{2} E}{9 \pi \varepsilon_{0} m^{4} c^{5}}
$$

which means that they take over at high enough energies. Equating Eq. (2) with Eq. (3), one obtains

$$
E_{\max } \propto \frac{m^{2}}{Z^{3 / 2}} \sqrt{\frac{\eta}{B}} \propto \sqrt{A} \sqrt{\frac{\eta}{B}}
$$

where in the latter step $m \propto A \propto Z$ was assumed, which is a good approximation for elements heavier than hydrogen. For hydrogen and $\eta=1$, the region where synchrotron losses dominate is on the rhs of the dashed line in the upper right panel of Fig. 2; that is, for shock acceleration and a moderately efficient acceleration, this region cannot be reached. We will consider the impact of this theoretical 

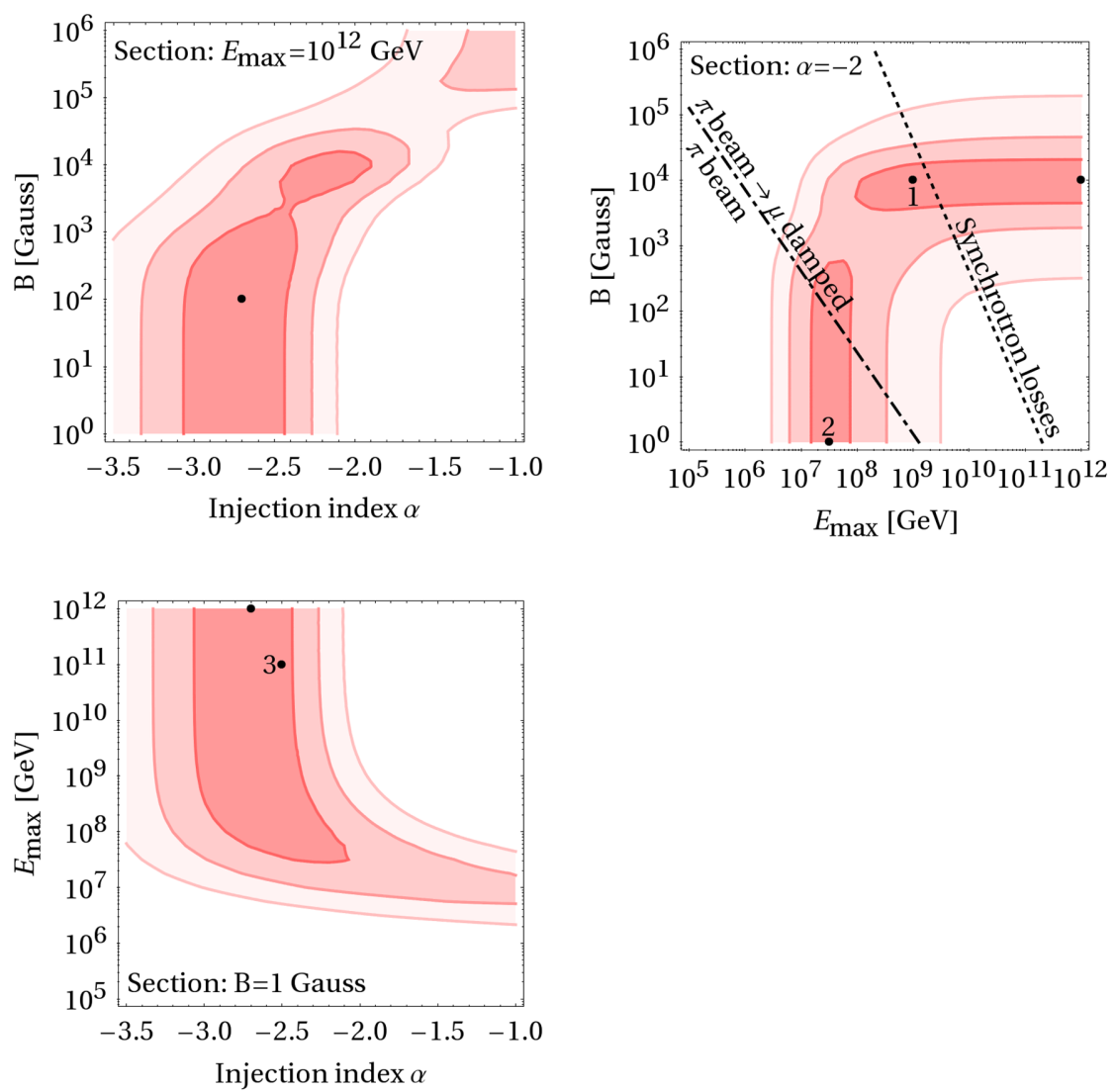

FIG. 2 (color online). Allowed fit regions to neutrino data at $1 \sigma, 2 \sigma$, and $3 \sigma$ (two d.o.f.) in a three-parameter $\left(\alpha, B, E_{\max }\right)$ model for protons only, including the full data set. The filled contours represent sections, i.e., the third (not shown) parameter in each panel is fixed to the depicted value. The overall minimum is shown as a dot, and three additional test points are marked as well (see Fig. 3). The lines in the upper right panel are discussed in the main text.

constraint on the fit below. Note that we do not assume relativistic boosting here. For example, for gamma-ray bursts $E_{\max } \simeq 10^{9} \mathrm{GeV}$ can be reached for $100 \mathrm{kG}$ in the shock rest frame, which translates into $E_{\max } \sim 10^{11} \mathrm{GeV}$ in the observer's frame. Note that an additional constraint comes from the size of the acceleration region (the Hillas criterion), which is however difficult to interpret for highly relativistic sources due to relativistic length contraction and energy boosting. We do not explicitly discuss this constraint here as it involves another parameter (the size of the acceleration region); this could be interesting for the interpretation of the signal in terms of specific source classes, but it only adds limited new information to our generic fit.

So what kinds of options do we have for describing the data? In order to illustrate this, three test points in the $1 \sigma$ region are marked in Fig. 2. We show the obtained best-fit spectra for these test points and electron neutrinos in the upper panel of Fig. 3. Furthermore, in the lower panel we show the flavor ratio of muon neutrinos to electron and tau neutrinos at the detector, which corresponds to the ratio between the induced muon tracks and cascades (without efficiencies). We can identify three options:
Point 1. High $E_{\max }$ values are allowed, together with strong magnetic fields. The magnetic fields lead to a cutoff and some characteristic wiggles in the spectrum, which come with a change of the flavor composition at $\mathrm{PeV}$ energies from a pion-beam to a muon-damped source; see the lower panel of Fig. 3.

Point 2. For small values of $B$, the cutoff can be achieved by an appropriate maximal proton energy, as discussed above.

Point 3. Alternatively, a soft enough spectrum can describe data for small $B$ and large $E_{\max }$; see also Ref. [41].

We will discuss Point 4 in the next section. Note that in all cases, the normalization (which is a result of the fit) is about $1.5 \times 10^{-8} \mathrm{GeV} \mathrm{cm}^{-2} \mathrm{~s}^{-1} \mathrm{sr}^{-1}$ at $30 \mathrm{TeV}$.

It is of course interesting to discuss what this information tells us about the sources. Point 1 corresponds to sources with strong magnetic fields, such as low-luminosity gammaray bursts [42], "chocked" gamma-ray bursts [43-45], or (extra-Galactic) microquasars or pulsars. Point 2 may 

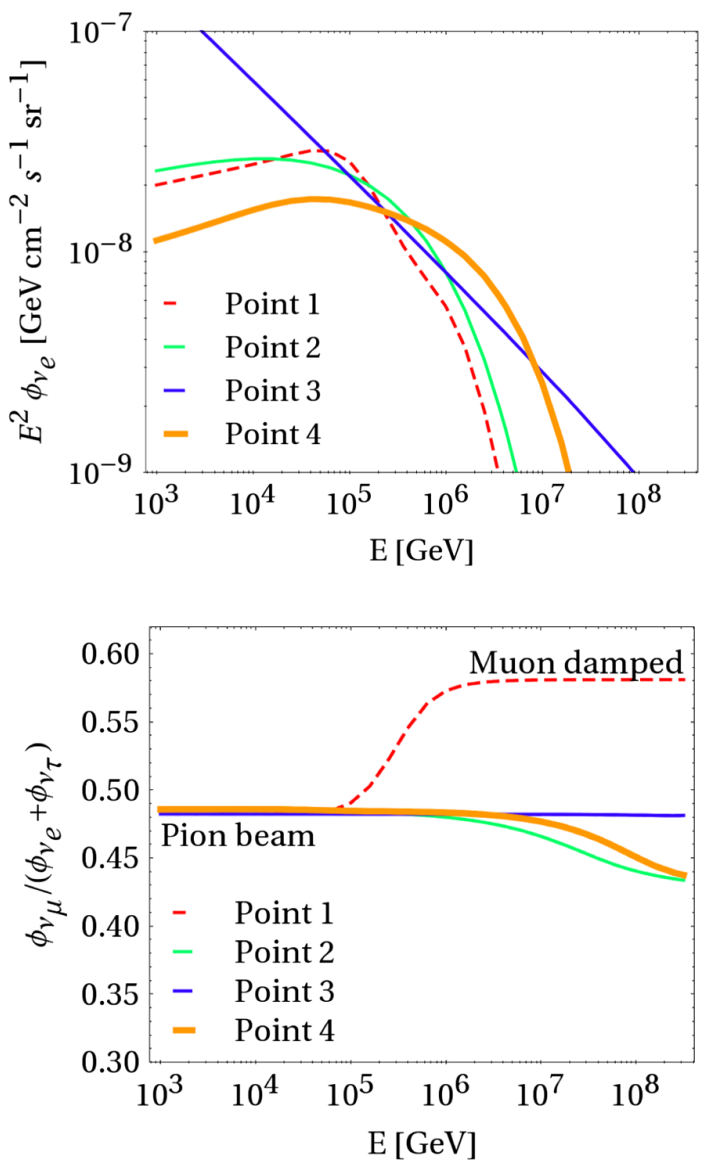

FIG. 3 (color online). Spectra (upper panel) and flavor composition at the detector (lower panel) corresponding to the points 1 to 3 marked in Fig. 2, where the normalization represents the best fit to IceCube data. Point 1 refers to $\alpha=-2, E_{\max }=$ $10^{9} \mathrm{GeV}$, and $B=10^{4} \mathrm{G}$; point 2 referes to $\alpha=-2$, $E_{\max }=10^{7.5} \mathrm{GeV}$, and $B \lesssim 1 \mathrm{G}$; and point 3 refers to $\alpha=$ $-2.5, E_{\max }=10^{11} \mathrm{GeV}$, and $B \lesssim 1 \mathrm{G}$. Point 4 refers to the heavier composition model, with $\alpha=-2, E_{\max }=10^{10.1} \mathrm{GeV}$, $B \lesssim 1 \mathrm{G}$, and $\beta=0.4$; see Fig. 5 .

correspond to starburst galaxies [22], galaxy clusters/groups [37], or radio galaxies [46]. And Point 3 may come from any extra-Galactic population, where the main challenge is to accommodate $\alpha \ll-2.2$ with the theory of Fermi acceleration. One possibility is the effect of turbulence on Fermi shock acceleration, which may cause such effects [47]; another possibility is that the overall spectral index comes from convoluting a harder spectrum with an appropriate luminosity distribution function [48].

Maybe even more interesting is the conceptual question of whether these neutrinos can come from the sources as the UHECRs. Considering Point 1 -which takes into account the synchrotron loss constraint in Fig. 2-the maximal energy can only be high enough to reach the UHECR range $E>10^{10} \mathrm{GeV}$ if large Lorentz boosts are involved. Point 2, on the other hand, cannot be accommodated within the UHECR paradigm, because $E_{\max }$ is too low. For Point 3, the discussion is much more complicated: while it can in principle be accommodated within the UHECR paradigm, the soft spectrum tends to lead to neutrino overproduction at $\mathrm{PeV}$ energies if one normalizes the UHECR range to normalization. This is discussed for gamma-ray bursts in Refs. [49,50], and, in a more generic context, in Ref. [51]. However, a model-independent "proof" seems more difficult.

In Fig. 4, we take multiparameter correlations into account; that is, we show the projections including the minimization of the parameter not shown in each panel. This increases the fit regions dramatically. On the other hand, we include the Murase-Ahlers-Lacki bound, which leads to $\alpha \gtrsim-2.2$ and the synchrotron loss constraint, explicitly shown in the upper right panel. These theoretical constraints reduce the size of the fit regions. The main result, which can be read from the left panels, is that spectral indices compatible with Fermi acceleration are preferred in combination with a cutoff of the maximal proton energy, whereas a wide range of magnetic fields are possible. The best fit at $\alpha \simeq 2$ and $E_{\max } \simeq 210^{9} \mathrm{GeV}$ points toward conventional scenarios of Fermi shock acceleration together with a maximal proton energy cutoff compatible with the ankle of the cosmic-ray spectrum observed in our Galaxy. It is therefore plausible that the neutrinos are produced under similar conditions, such as in starburst galaxies.

\section{NUCLEI-MATTER INTERACTIONS AND THE UHECR PARADIGM}

Adding the composition to the parameters of the model increases the complexity to a level such that no meaningful information can be obtained from current data due to limited statistics. We therefore focus on a key issue the proton composition has not been successfully described: can the potential cutoff at $\mathrm{PeV}$ energies be reconciled with the UHECR paradigm, taking into account that the composition could be as heavy as iron at the highest energies [17]?

Let us assume that the magnetic fields are small enough that magnetic-field effects on the secondaries are negligible. This is a necessary condition so that the maximal energies are not suppressed by synchrotron losses in our standard scenario [for example, if one applies Eq. (4) to the dashed curve in Fig. 2 for $E_{\max }=10^{11} \mathrm{GeV}$ ]. Let us furthermore assume that $\alpha \simeq-2$ in consistency with the argument in Ref. [51], and that $A\left(E_{\max }\right)=56$ (iron). We then assume that the maximal energy is element dependent, which leads to an energy-dependent composition parametrized as

$$
A(E)=\max \left(1,56 \times\left(\frac{E}{E_{\max }}\right)^{\beta}\right),
$$

i.e., $A(E) \geq 1$ and $A\left(E_{\max }\right)=56$. The coefficient $\beta$ describes how $A$ scales with energy, and we treat it as a continuous parameter. However, it is useful to consider a few examples: 

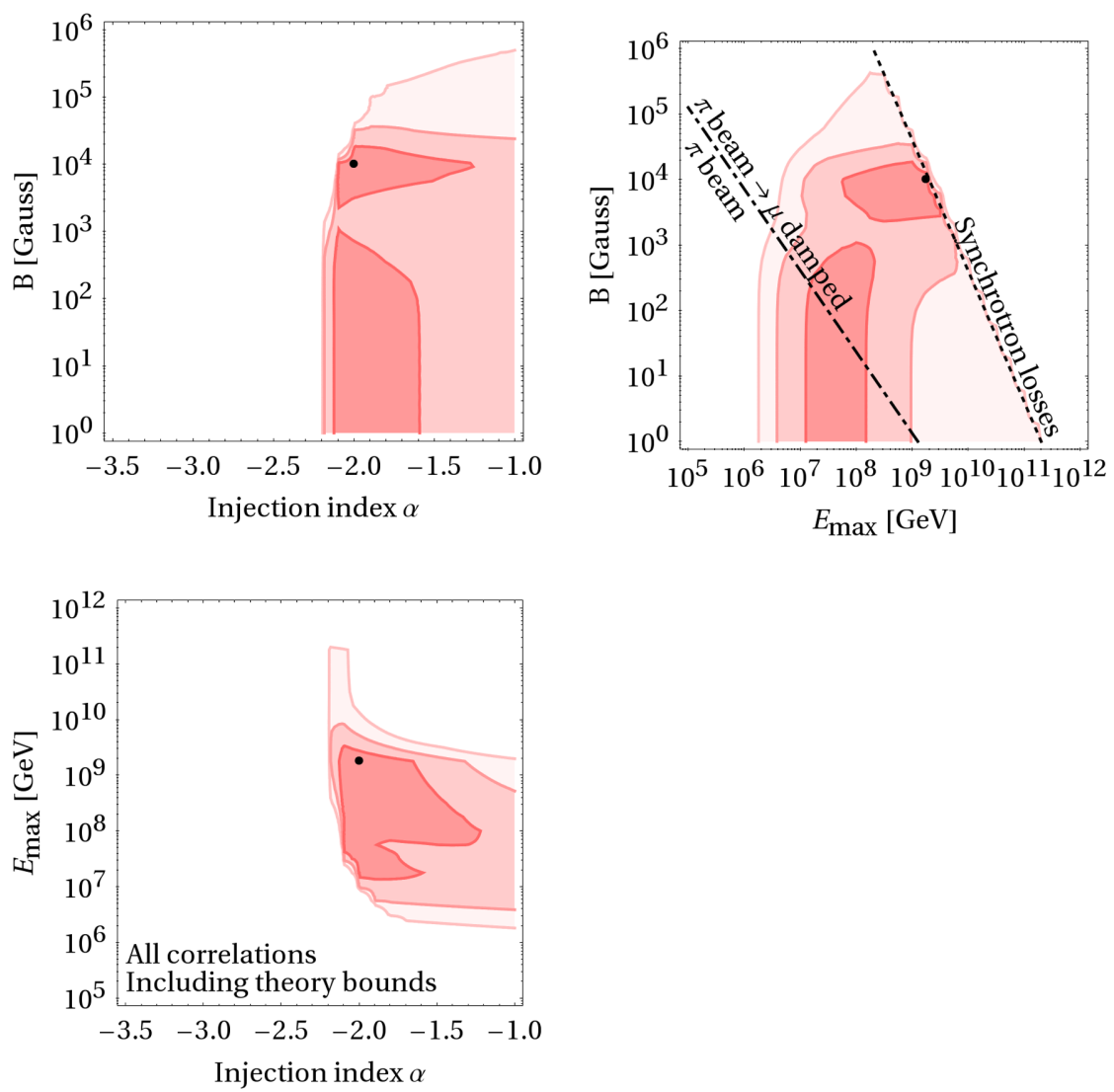

FIG. 4 (color online). Allowed fit regions to neutrino data at $1 \sigma, 2 \sigma$, and $3 \sigma$ (two d.o.f.) in a three-parameter $\left(\alpha, B, E_{\max }\right)$ model for protons only. Here the full parameter degeneracy is taken into account, i.e., the $\chi^{2}$ is minimized over the third parameter in each panel. Here theoretical exclusion limits are included as $\chi^{2}$ penalties, i.e., the Murase-Ahlers-Lacki bound on $\gamma$-ray observations and the region that is unreachable due to synchrotron losses dominating the maximal proton energy as a cutoff.

Rigidity scaling: This is the most often used approach, also known as the necessary condition formulated by Hillas [40]: the Larmor radius has to be smaller than the acceleration region. This can also be described using Eq. (2) as $t_{\text {acc }}^{-1}=t_{\text {lim }}^{-1} \simeq c / R$, where $R$ is the size of the region and $t_{\lim }^{-1}$ is the limiting time scale determined by the size of the region (typically the dynamical time scale, escape time scale, or adiabatic cooling time scale). As a consequence, $E / Z$ (the rigidity) is constant for constant $B$ and $R$, which means that higher energies can be reached for higher charges. Since $Z \sim A / 2$, one has $\beta \simeq 1$.

Synchrotron-loss-dominated $E_{\max }$ : From Eq. (4) describing a shock acceleration scenario, we can immediately see that $A \propto E^{2}$, i.e., $\beta=2$, if the maximal energy is limited by synchrotron losses.

It is generally difficult to obtain $\beta<1$ unless the time scale constraining the maximal energy slightly drops with energy and does not scale with rigidity. This may be achieved in scenarios where, e.g., photo-disintegration dominates the highest energies [42]. However, we note that the composition has been observed to be light at $10^{9} \mathrm{GeV}$ [17,52], which gives a constraint $E_{\max }(\beta)$ that can be easily derived from Eq. (5) using $A\left(10^{9} \mathrm{GeV}\right)=1$.

The current best-fit region in terms of $\beta$ and $E_{\max }$ is shown in Fig. 5 for fixed $\alpha=-2$. It is clear from the figure that $E_{\max } \gg 10^{10} \mathrm{GeV}$ (which is required to describe UHECR observations) implies that $\beta<1$. Extremely high energies are allowed for $0.05 \lesssim \beta \lesssim 0.35$, which requires unconventional assumptions for the acceleration-radiation scenarios, as discussed above. This scenario requires neither strong magnetic fields nor a spectral index softer than $\alpha=-2$; the cutoff is instead produced by a change of the composition. Note that in this case neutrino data can be used to infer the acceleration of the heavier elements itself, or to model the injected UHECR composition from the sources in propagation codes. The spectrum corresponding to test point 4 is also shown in Fig. 3 (upper panel). It peaks at somewhat higher energies than the other spectra. It is probably noteworthy that, because $\alpha=-2$, there are no issues with the MAL bound in this case, as we have explicitly tested. However, this scenario is in slight tension with the observed light composition at $10^{9} \mathrm{GeV}$ [which is for protons (extreme case)], shown as a dotted curve in Fig. 5. If slightly heavier compositions are admitted at 


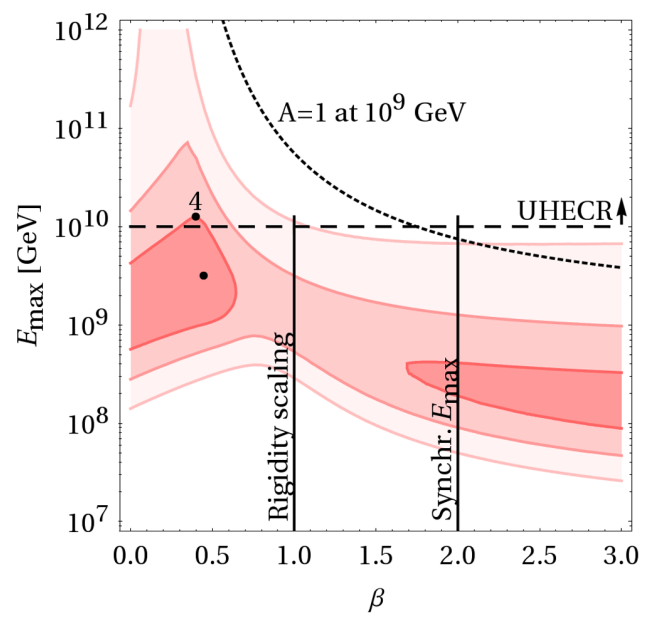

FIG. 5 (color online). Allowed fit region to neutrino data at $1 \sigma$, $2 \sigma$, and $3 \sigma$ (two d.o.f., filled contours) as a function of $\beta$ and $E_{\max }$ for $\alpha=-2$, and with $B$ small enough such that magnetic-field effects on the secondaries can be neglected. Here the composition is chosen to be iron at the highest energy $E_{\max }$, and the composition is assumed to be energy dependent with $A(E)=\max \left(1,56 \times\left(E / E_{\max }\right)^{\beta}\right)$. The vertical lines correspond to different acceleration scenarios, as discussed in the main text. If the composition is to be dominated by protons at $10^{9} \mathrm{GeV}$, the dotted curve has to be matched.

$10^{9} \mathrm{GeV}$ such as helium, the dotted curve would move closer to the fit contours.

\section{FUTURE EXPECTATIONS FOR ICECUBE PERFORMANCE}

We finally discuss what can be learned from future upgrades of IceCube. As an example shown in Fig. 6, let us

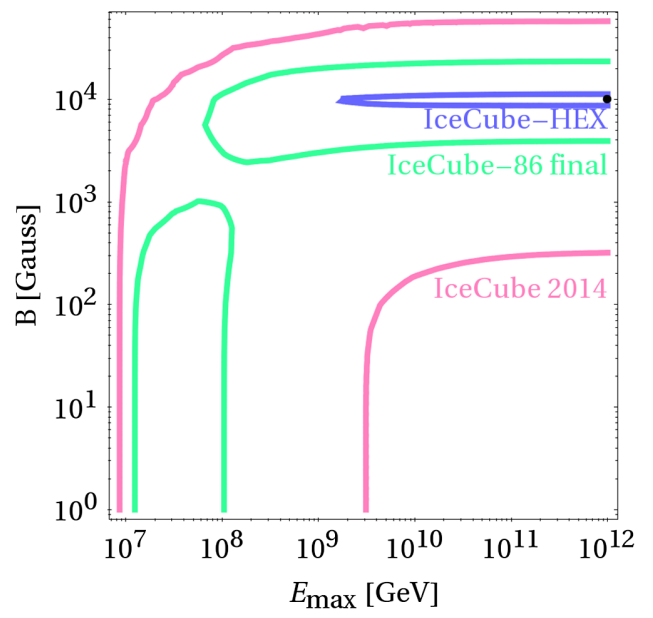

FIG. 6 (color online). Extrapolation of the $3 \sigma$ allowed region as a function of $E_{\max }$ and $B$ (the section is for protons and $\alpha=-2$, corresponding to the upper right panel of Fig. 2, but including the MAL bound). "IceCube 2014" corresponds to current data (37 events), "IceCube-86 final" to four times the current exposure, and "IceCube-HEX" to a possible high-energy extension with 40 times the current exposure (a factor of 10 larger mass operated over about a decade). The best fit is marked by a dot. choose the $3 \sigma$ allowed region as a function of $E_{\max }$ and $B$ corresponding to the upper right panel of Fig. 2 (but including the MAL bound). The outer (red) curve shows the constraint from current data, the middle green region shows the expected result from IceCube- 86 over about a decade (four times the current exposure), and the blue region shows the expected result from a future HEX with about ten times the capacity of IceCube, operated over about a decade. This figure illustrates that while better information will be available in a few years with upcoming data, high precision will require an upgrade of IceCube. In this particular case IceCube-86 cannot discriminate between a cutoff from magnetic-field effects or a cutoff in the proton spectrum, corresponding to test points 1 and 2. However, the precision in IceCube-HEX will even allow one to exploit the transition in the flavor composition expected by magnetic-field effects, and discriminate between these regions. This is evident from the event rates. Consider, e.g., Point 1 and the muon track and cascade bins between 1-2 PeV. For current statistics, the expectations are 0.6 tracks and 0.9 cascades for these bins, whereas for IceCube-HEX the expectations are 24 muon tracks and 37 cascades. This leads to a statistical relative error of about $1 / \sqrt{24} \simeq 20 \%$. The muon track-to-shower ratio is increased by about $20 \%$ in the muon-damped case (coinciding with this energy range for the chosen test point) compared to the pion-beam case after flavor mixing (see lower panel of Fig. 3, between 1-2 PeV), which means that the statistics between muon tracks and showers becomes meaningful. This is a significant qualitative advance compared to the full-statistics IceCube- 86 analysis.

\section{SUMMARY AND DISCUSSION}

We have studied the interpretation of IceCube data in the production scenario of nuclei-matter interactions, for which the neutrino spectrum follows the nonthermal spectrum of the nuclei. Compared to earlier studies, we have taken into account possible magnetic-field effects on the secondary muons and pions and the composition of the accelerated nuclei. In particular, we have focused on the reproduction of the spectrum, where the flavor composition (cascades versus muon tracks) has been implied as well. We have essentially identified four different options for the initial spectrum of the protons/nuclei to reproduce current data, which can all avoid the overproduction of events beyond a few PeV:

(1) An unbroken power law with $\alpha \sim-2$ and magnetic fields $B \sim 10^{4} \mathrm{G}$ in the source, leading to magneticfield effects on the secondary muons and pions. This may be realized in certain populations of gamma-ray bursts or (extra-Galactic) microquasars or pulsars.

(2) A power law with $\alpha \sim-2$ with a break or cutoff at about $10^{7}-10^{8} \mathrm{GeV}$ (for protons). This option may be favorable for models of starburst galaxies, galaxy clusters/groups, or radio galaxies. 
(3) An unbroken power law significantly softer than $E^{-2}$, where we find best-fit values for $\alpha$ between -2.7 and -2.3 depending on the analysis range and atmospheric background model. This option is the simplest one, (and was also mentioned in the recent IceCube three-year analysis [3]), where we typically find softer indices because of the larger energy range being analyzed. A major drawback has been pointed out by MAL [37]: the spectrum consequently exceeds the isotropic gamma-ray background at lower energies, unless a low-energy break is introduced.

(4) An unbroken power law with $\alpha \sim-2$ and a flat enough change of the composition of the nonthermal spectra in the source from lighter to heavier elements at the highest energies, which can potentially be as high as $10^{12} \mathrm{GeV}$.

While options 1 to 3 have been identified in similar forms in the literature, option 4 is entirely new. Regarding options 1 and 2, we note that similar possibilities are obtained for photohadronic interactions that produce neutrinos, for which the spectral shape is determined by both the nuclei and target photon spectra; see Ref. [7].

We have been particularly interested in which of the above options can be reconciled with the question of whether the observed neutrinos stem from the sources of the UHECRs; see also the discussion in Refs. [53,54]. Option 2 is obviously incompatible with this assumption if the break or cutoff can also be found in the escaping proton spectrum injected into the intergalatic medium. Exceptions could be scenarios with energy-dependent diffusive escape, because in that case the steady proton spectrum (responsible for neutrino production) can have a break, whereas the escaping proton spectrum can be different; see e.g., Ref. [53] for hypernova remnants. Option 1 faces the problem that synchrotron losses typically limit the maximal proton energy, and do not allow for high enough maximal energies (unless strong Lorentz boosts are involved, such as in gamma-ray bursts). Option 3, on the other hand, tends to lead to an overproduction of neutrinos at $\mathrm{PeV}$ energies exceeding the current IceCube observations when normalized to the UHECR observations (see Ref. [51] for a generic discussion) while also violating the MAL bound. We have therefore identified option 4 as the most promising one, especially in light of recent Auger observations pointing towards a heavier composition at the highest energies. It is generally compatible with both the UHECR paradigm - as the acceleration energies can be high enough to describe observations - and the MAL bound. In fact, we have demonstrated that neutrino data can be used to test the acceleration mechanism in this scenario. On the other hand, the results from neutrino observations may serve as an input for the UHECR injection at the sources in UHECR propagation models. However, this scenario is in slight tension with a very light composition at $10^{9} \mathrm{GeV}$.

Finally, we have pointed out that future precision measurements will require significant volume upgrades, such as a high-energy extension of IceCube or KM3NeT in the Mediterranean. While this statement is most certainly generally true, we have shown that the current IceCube experiment will not be sufficient to exploit the flavor information from muon tracks versus cascades in a statistically meaningful manner, in order to discriminate between pion-beam and muon-damped sources. The reason for this is that the flavor transition is expected at high enough energies to describe the cutoff at $\mathrm{PeV}$ energies, where the event rates are very low. However, a significantly larger (about a factor of 10) volume upgrade would allow for a discrimination between options 1 and 2, where the flavor information will be the qualitatively new ingredient. It is therefore important to optimize an upgrade in a way that preserves the flavor or topology identification capability.

\section{ACKNOWLEDGMENTS}

I would like to thank Markus Ackermann, Sergio Palomares-Ruiz, Karl Mannheim, Lars Mohrmann, Julia Tjus, Xiang-Yu Wang, and Nathan Whitehorn for useful discussions and comments on aspects of this work, and Lars Mohrmann for reading the manuscript. I would like to acknowledge support from DFG grants WI 2639/3-1 and WI 2639/4-1, the "Helmholtz Alliance for Astroparticle Physics HAP," funded by the Initiative and Networking fund of the Helmholtz Association. I would also like to acknowledge support from the Nordita program "News in neutrino physics" from April 21-May 2, 2014, where parts of this work were carried out.
[1] M. Aartsen et al. (IceCube Collaboration), Phys. Rev. Lett. 111, 021103 (2013).

[2] M. Aartsen et al. (IceCube), Science 342, 1242856 (2013).

[3] M. Aartsen et al. (IceCube Collaboration), Phys. Rev. Lett. 113, 101101 (2014).
[4] M. Ahlers and F. Halzen, Phys. Rev. D 90, 043005 (2014).

[5] U. F. Katz, Nucl. Instrum. Methods Phys. Res., Sect. A 567, 457 (2006).

[6] L. A. Anchordoqui et al., J. High Energy Astrophys. 1-2, 1 (2014). 
[7] W. Winter, Phys. Rev. D 88, 083007 (2013).

[8] M. Kachelriess, S. Ostapchenko, and R. Tomas, Phys. Rev. D 77, 023007 (2008).

[9] P. Lipari, M. Lusignoli, and D. Meloni, Phys. Rev. D 75, 123005 (2007).

[10] S. Hümmer, M. Maltoni, W. Winter, and C. Yaguna, Astropart. Phys. 34, 205 (2010).

[11] T. Kashti and E. Waxman, Phys. Rev. Lett. 95, 181101 (2005).

[12] K. Murase and S. Nagataki, Phys. Rev. D 73, 063002 (2006).

[13] P. Baerwald, S. Hümmer, and W. Winter, Astropart. Phys. 35, 508 (2012).

[14] M. M. Reynoso and G. E. Romero, Astron. Astrophys. 493, 1 (2009).

[15] P. Baerwald and D. Guetta, Astrophys. J. 773, 159 (2013).

[16] W. Winter, Adv. High Energy Phys. 2012, 586413 (2012).

[17] J. Abraham et al. (Pierre Auger Observatory Collaboration), Phys. Rev. Lett. 104, 091101 (2010).

[18] S. Kelner, F. A. Aharonian, and V. Bugayov, Phys. Rev. D 74, 034018 (2006).

[19] J. C. Joshi, W. Winter, and N. Gupta, Mon. Not. R. Astron. Soc. 439, 3414 (2014).

[20] L. A. Anchordoqui, J. F. Beacom, H. Goldberg, S. PalomaresRuiz, and T. J. Weiler, Phys. Rev. D 75, 063001 (2007).

[21] M. Gonzalez-Garcia, M. Maltoni, J. Salvado, and T. Schwetz, J. High Energy Phys. 12 (2012) 123.

[22] A. Loeb and E. Waxman, J. Cosmol. Astropart. Phys. 05 (2006) 003.

[23] L. A. Anchordoqui, T. C. Paul, L. H. M. da Silva, D. F. Torres, and B. J. Vlcek, Phys. Rev. D 89, 127304 (2014).

[24] I. Tamborra, S. Ando, and K. Murase, J. Cosmol. Astropart. Phys. 09 (2014) 043.

[25] X.-C. Chang and X.-Y. Wang, Astrophys. J. 793, 131 (2014).

[26] S. Hümmer, P. Baerwald, and W. Winter, Phys. Rev. Lett. 108, 231101 (2012).

[27] S. R. Klein, R. Mikkelsen, and J. K. B. Tjus, Astrophys. J. 779, 106 (2013).

[28] W. Winter, J. B. Tjus, and S. R. Klein, Astron. Astrophys. 569, A58 (2014).

[29] M. M. Reynoso, Astron. Astrophys. 564, A74 (2014).

[30] A. M. Hopkins and J. F. Beacom, Astrophys. J. 651, 142 (2006).

[31] R. Laha, J. F. Beacom, B. Dasgupta, S. Horiuchi, and K. Murase, Phys. Rev. D 88, 043009 (2013).
[32] R. Abbasi et al. (IceCube Collaboration), Phys. Rev. D 83, 012001 (2011).

[33] R. Abbasi et al. (IceCube Collaboration), Phys. Rev. D 84, 082001 (2011).

[34] T. Sinegovskaya, E. Ogorodnikova, and S. Sinegovsky, arXiv:1306.5907.

[35] O. Mena, S. Palomares-Ruiz, and A. C. Vincent, Phys. Rev. Lett. 113, 091103 (2014).

[36] C.-Y. Chen, P. S. B. Dev, and A. Soni, Phys. Rev. D 89, 033012 (2014).

[37] K. Murase, M. Ahlers, and B. C. Lacki, Phys. Rev. D 88, 121301 (2013).

[38] A. Abdo et al. (Fermi-LAT collaboration), Phys. Rev. Lett. 104, 101101 (2010); M. Ackermann, in Proceedings of the Fourth International Fermi Symposium, Monterey, CA, USA, 238, 2012 (to be published).

[39] J. G. Learned and S. Pakvasa, Astropart. Phys. 3, 267 (1995).

[40] A. M. Hillas, Annu. Rev. Astron. Astrophys. 22, 425 (1984).

[41] L. A. Anchordoqui, H. Goldberg, M. H. Lynch, A. V. Olinto, T. C. Paul, and T.J. Weiler, Phys. Rev. D 89, 083003 (2014).

[42] K. Murase, K. Ioka, S. Nagataki, and T. Nakamura, Phys. Rev. D 78, 023005 (2008).

[43] S. Razzaque, P. Meszaros, and E. Waxman, Phys. Rev. Lett. 93, 181101 (2004).

[44] S. Ando and J. F. Beacom, Phys. Rev. Lett. 95, 061103 (2005).

[45] S. Razzaque, P. Meszaros, and E. Waxman, Mod. Phys. Lett. A 20, 2351 (2005).

[46] J. B. Tjus, B. Eichmann, F. Halzen, A. Kheirandish, and S. Saba, Phys. Rev. D 89, 123005 (2014).

[47] M. Lemoine, G. Pelletier, and B. Revenu, Astrophys. J. 645, L129 (2006).

[48] M. Kachelriess and D. V. Semikoz, Phys. Lett. B 634, 143 (2006).

[49] M. Ahlers, M. Gonzalez-Garcia, and F. Halzen, Astropart. Phys. 35, 87 (2011).

[50] P. Baerwald, M. Bustamante, and W. Winter, Astropart. Phys. 62, 66 (2015).

[51] B. Katz, E. Waxman, T. Thompson, and A. Loeb, arXiv:1311.0287.

[52] R. Abbasi et al. (HiRes Collaboration), Phys. Rev. Lett. 104, 161101 (2010).

[53] R.-Y. Liu, X.-Y. Wang, S. Inoue, R. Crocker, and F. Aharonian, Phys. Rev. D 89, 083004 (2014).

[54] M. D. Kistler, T. Stanev, and H. Yuksel, arXiv:1301.1703. 